Artículo

\title{
Análisis y priorización de sistemas de producción asociadas al café y aguacate
}

Diana Ayala Montejo

Eduardo Valdés Velarde

José Luis Romo Lozano ${ }^{\S}$

${ }^{1}$ Universidad Autónoma Chapingo. Carretera México-Texcoco km 38.5, Chapingo, Texcoco, Estado de México, México. CP. 56230. (diayalamontejo@gmail.com; valdevela@gmail.com).

${ }^{\S}$ Autor para correspondencia: jlromo@aya.yale.edu.

\section{Resumen}

La presión por el cambio a monocultivos de aguacate en los sistemas tradicionales de café en el estado de Veracruz puede generar consecuencias, puesto que este cultivo busca maximizar la rentabilidad financiera sin considerar la sostenibilidad de empleo, gestión, adaptar e implementar tecnologías ambientalmente amigables, capacidad de inversión y servicios ecosistémicos. El objetivo fue analizar y priorizar distintos sistemas de producción asociados al cultivo de café y aguacate, mediante dos escenarios: financiero (EF) y multicriterio (EMC). En EF se incluyeron medidas monetarias, con las que se calculó la relación beneficio costo (RBC); en el EMC, además de RBC se incorporaron los criterios de necesidad de inversión, empleo, gestión, carbono y nitrógeno en biomasa vegetal, biomasa y diversidad de macrofauna edáfica, analizados con el método PROMETHEE; a través, del cual se generó un ordenamiento de priorización. Los análisis se realizaron en cinco sistemas: aguacate monocultivo (SAM), aguacate café (SAC), cafetal renovado (SCR); cafetal con limpiezas severas (SCL) y cafetal abandonado (SCA), con información del ciclo 2018-2019. En EF el SAM resultó con la RBC muy por encima de los demás (2.43). El EMC priorizo a los SCR, SAM y SAC en las tres primeras posiciones de la clasificación. Los análisis fundamentan la amenaza de los SAM para desplazar a los cafetales, por lo que se mantiene el reto de diseñar y poner en práctica políticas públicas que valoren las variables sociales y ambientales, y consolidar así SAC en una mejor posición que permitan mantener el cultivo de café.

Palabras clave: agroforestería, diversidad productiva, multicriterio, PROMETHEE, sostenibilidad.

Recibido: enero de 2021

Aceptado: marzo de 2021 


\section{Introducción}

Actualmente en Veracruz los productores tienden a optar por la plantación de aguacate, puesto que su producción genera ganancias netas hasta de $4695.9 .00 \$ \mathrm{t}^{-1}$, con una producción de $100 \mathrm{~kg}$ por árbol y una densidad de siembra de 100 plantas ha $^{-1}$ (Franco et al. 2018), valor que superó los 2 $630.00 \$ \mathrm{ha}^{-1}$ de ingresos netos generados en cafetales afectados por roya con un rendimiento de $4.5 \mathrm{t} \mathrm{ha}^{-1}$, valorizado a $5000.00 \$ \mathrm{t}^{-1}$, considerando un costo de mantenimiento y cosecha de 19 $870.00 \$$ ha $^{-1}$ (FIRA, 2016).

En Veracruz domina la producción de café cultivado bajo sombra diversificada (SánchezHernández et al., 2018), estos sistemas son importantes por: la riqueza de su composición florística (Sánchez et al., 2017), la baja inversión en la producción; y el aporte al almacenamiento de carbono y nitrógeno. Sin embargo, esta actividad genera bajos rendimientos para los caficultores de Veracruz. Este problema que se atribuye a las variaciones climáticas que han generado la proliferación de roya, la cual afecta el rendimiento de los cafetos (Granados et al., 2014), lo que los hace vulnerables a la expansión del cultivo de aguacate como monocultivo.

El cambio de sistemas tradicionales de café por sistemas modernos, como el cultivo de aguacate en Veracruz, puede generar consecuencias negativas en la sostenibilidad social y ambiental, pues estos sistemas buscan maximizar la rentabilidad financiera sin considerar otros factores fundamentales de carácter social como la capacidad de inversión del pequeño productor y de carácter ambiental como el potencial para mantener los almacenes de carbono cuando se manejan cafetales diversificados (Ayala et al., 2020). El tema de los sistemas agroforestales (SAF) con café ha sido abordado desde diversos enfoques, existen estudios que utilizan las variables socioeconómicas para caracterizar a los productores de café y su relación con su condición actual, recursos locales, acceso a servicios básicos y manejo, con el fin de identificar eslabones de valor (Aguirre et al., 2016; Leiva et al., 2017; Pertuz y Pérez, 2017).

Del mismo modo, Escalante y Somarriba (2001), indican que las variables que determinan la tipología de un SAF con café son los costos de fertilización, fungicidas y mano de obra para mantenimiento, y estás son directamente proporcionales a la composición funcional del dosel de sombra y por tanto son importantes para clasificar a los SAF. Por otra parte, Dussán et al. (2006) identificaron que la estructura de los SAF, su producción y productividad dependen de las variables nivel de educación y acceso a la tecnología. Estos estudios evidencian la importancia de las variables socioeconómicas para caracterizar a los SAF con café, determinar su tipología, valorar la importancia de la diversificación productiva y la aplicación y adopción de tecnología; sin embargo, aún se desconocen la rentabilidad y las variaciones de las variables socioeconómicas y ambientales de los cafetales asociados con aguacate.

El análisis integrado de estos factores para los sistemas de café asociados a aguacate permite caracterizarlos bajo un mismo patrón de comparación y analizar sus relaciones, lo que contribuye a plantear estrategias de diversificación productiva que integren los servicios ambientales con las variables socioeconómicas y una producción sostenible. Bajo este enfoque, el presente trabajo tiene como objetivo analizar y priorizar las opciones de producción asociadas a los cultivos de café y aguacate en Huatusco, Veracruz, México. 
El análisis y priorización se proponen a la luz de escenarios que consideran, además del criterio privado, aspectos de interés público con inclusión de variables que permitan mensurar la necesidad de inversión, empleo, gestión para innovar, contenidos de carbono y nitrógeno, la diversidad del suelo (biomasa y diversidad de la macrofauna edáfica), que aportan al bienestar social y ambiental.

La mayoría de los problemas que implican decisiones relacionadas con priorización, clasificación y elección, involucran varios criterios, los cuales se pueden abordar de manera integrada mediante métodos que se agrupan en el análisis multicriterio (AMC). El AMC ha sido utilizado ampliamente en el sector agrícola como una herramienta que permite organizar los diversos criterios y alternativas, además de apoyar estratégicamente una variedad de decisiones desafiantes de los agricultores y los encargados de formular políticas (Berbel et al., 2018).

Existen estudios de análisis multicriterio que apoyan la toma de decisiones de gestión de regiones agrícolas enteras (Bournaris et al., 2009; Manos et al., 2010), impactos y escenarios de políticas (Manos et al., 2006, 2013; Riesgo y Gómez, 2006; Bournaris y Manos, 2012; Bournaris et al., 2014), disparidades en las zonas rurales (Popescu y Bara, 2015) producción de biomasa (Tziolas et al., 2017; Caprara y Martelli, 2016; Kylili et al., 2016), estrategias para conservar la biodiversidad (Moffett y Sarkar 2006), decisión sobre la ubicación del proyecto de cogeneración de biomasa agroforestal (Wu et al., 2019), entre otros.

Estos estudios evidencian que el AMC ha permitido integrar múltiples aspectos bajo un enfoque holístico que contribuyen a la toma de decisiones, puesto que esta herramienta permite evaluar los impactos, la selección de criterios, la ponderación y la agregación de indicadores (Finkbeiner et al., 2010). Sin embargo, la literatura aún no reporta casos de aplicaciones sobre decisiones de sistemas agroforestales con diversificación productiva con café y aguacate que permitan la sostenibilidad de los cafetales afectados por la roya a escala de pequeño productor.

En los últimos años el análisis multicriterio se ha usado en diferentes ramas científicas (Maia et al., 2017) debido a su practicidad y utilidad de integración metodológica. Se han implementado varios métodos de AMC para evaluar la sostenibilidad ambiental, económica y social en la producción agrícola. Una de las distinciones entre las categorías de AMC se basa en el enfoque de agregación completa y el enfoque de superación, ya que los métodos interactivos (aproximación de prueba y error) no son aplicables en un análisis multivariado (Benoit y Rousseaux, 2003). Entre los métodos más utilizados, se menciona: Multi-Attribute Utility Theory (MAUT) (Lipuscek et al., 2010; Myllyviita et al., 2012); métodos de superación (PROMETHEE y ELECTRE) (Kralisch et al., 2013; Castellini et al., 2012); proceso de jerarquía analítica (AHP) (Dinh et al., 2009; De Luca et $a l ., 2015$ ); técnica de calificación de múltiples atributos simples (SMART) (Myllyviita et al., 2014); Visekriterijumska Optimizacija IKompromisno Resenje (VIKOR) (Falcone et al., 2016); técnica de orden preferencia similitud con solución ideal (TOPSIS) (Karklina et al., 2015).

La ventaja del AMC es que puede integrar aspectos subjetivos económicos, ambientales y sociales a la evaluación de manera objetiva (Myllyviita et al., 2014; De Luca et al., 2017), puesto que pueden normalizarse y ponderarse con la aplicación de diversos métodos como: ELECTRE, PROMETHEE y TOPSIS. La ponderación y normalización a través de estos métodos proporciona transparencia para realizar la interpretación, ya que la implementación sin las subetapas de normalización y ponderación podría revelar problemas para la agregación y la comparación de los resultados (Bengtsson y Steen, 2004). 


\section{Materiales y métodos}

El estudio se realizó en Huatusco Veracruz, México, donde se seleccionaron cinco casos de estudios con la metodología bola de nieve tradicional, estos casos corresponden a los tipos de manejo representativos de la zona: sistema de café con abandono del cultivo por 12 años (SCA), sistema de café renovado con variedades de café tolerantes a roya (SCR), sistema de café con podas y limpieza severas (SCL), sistema de aguacate con café (SAC) y sistema con aguacate como monocultivo (SAM).

Cada sistema fue caracterizado a través de entrevistas estructuradas donde se abordaron las variables económicas que corresponden a los costos de instalación, mantenimiento, insumos, cosecha, rendimiento, la calidad de cereza de café y calidad de fruto de aguacate en cada sistema. Para las variables sociales se consideró la cantidad de jornales para cada una de las actividades realizadas en los sistemas, las tecnologías instaladas y el número de capacitaciones en que participan anualmente, con el fin de valorizar las capacidades del pequeño productor para mantener y cambiar su sistema de producción. Las variables ambientales fueron el contenido de carbono y nitrógeno en biomasa vegetal, la biomasa y diversidad de macrofauna edáfica.

Estás variables permitieron obtener ocho criterios, agrupados en tres dimensiones: (1) financiera, que incluye la relación beneficio costo (RBC); (2) social, que contempla la necesidad de inversión (NI), Empleo (E) y gestión para innovar (G); y (3) ambiental, que considera el contenido de carbono en biomasa vegetal superior $(\mathrm{C})$, contenido de nitrógeno en biomasa vegetal inferior $(\mathrm{N})$, biomasa de macrofauna edáfica $(\mathrm{BM})$ y diversidad de macrofauna edáfica (DM). La metodología para determinar cada criterio se detalla en el Cuadro 1.

\section{El análisis de la información recabada se desarrolló en el marco de dos escenarios}

Análisis financiero, en el cual únicamente se considera la RBC, basado en medidas monetarias. Análisis multicriterio, en el cual además de la relación beneficio-costo, se integraron aspectos no monetarios considerados en las dimensiones social y ambiental. En este escenario, a cada una de las tres dimensiones se le asignó un mismo peso de importancia (33.33\%), quedando los pesos de los distintos criterios de la siguiente manera: RBC (33.33\%), NI (11.1\%), E (11.1\%), G (11.1\%), C (8.34\%), N (8.34\%), BM (8.34\%), DM (8.34\%). Se consideró el mismo peso para todos los criterios de acuerdo con el enfoque de sostenibilidad de los sistemas de producción (Hermann et al., 2007; Maia et al., 2017).

En el primer escenario se analizó el flujo de caja proyectado a un ciclo de producción de 30 años con una tasa de descuento de $10 \%$ para cada sistema, para luego determinar la relación beneficio costo con la información de los ingresos generados por cada producto del sistema y los costos de instalación, mantenimiento, producción y cosecha.

Todos estos valores fueron analizados en función de la tendencia de producción anual de cada cultivo del sistema y con el inicio de producción a partir del tercer año de sembrado. El valor de la tasa de descuento fue considerado de acuerdo con la metodología propuesta por Pérez y Garza (2013) para este tipo de sistemas. 
Para el análisis del segundo escenario, análisis multicriterio, se utilizó la información recabada en cada uno de los criterios, para cada uno de los sistemas (Cuadro 1), donde se destaca que el criterio NI es el único a minimizar, puesto que menor necesidad de inversión es mejor. En este escenario se aplicó el método PROMETHEE II, cuyo acrónimo proviene de sus siglas en inglés (Preference Ranking Organization for Enriched Evaluation). El método se basa en la relación de superación entre las alternativas tomando en cuenta los distintos valores que éstas tienen en cada criterio.

\section{Cuadro 1. Metodologías para determinar los valores de los criterios}

\begin{tabular}{|c|c|c|}
\hline Dimensión & Criterios & Metodología \\
\hline Financiera & $\begin{array}{l}\text { Relación beneficio costo } \\
\text { (RBC) }\end{array}$ & $\begin{array}{c}\text { Análisis financiero del flujo de caja, con una tasa de } \\
\text { descuento de } 10 \% \text { y una proyección de } 30 \text { años }\end{array}$ \\
\hline \multirow[t]{3}{*}{ Social } & $\begin{array}{l}\text { Necesidad de inversión } \\
\text { (NI) }\end{array}$ & $\begin{array}{c}\text { Monto de capital requerido para desarrollar el } \\
\text { proyecto productivo }\end{array}$ \\
\hline & Empleo $(\mathrm{E})$ & $\begin{array}{c}\text { Monitoreo de la cantidad de jornales utilizados para } \\
\text { el manejo y producción en cada sistema, proyectado } \\
\text { a un ciclo productivo de } 30 \text { años }\end{array}$ \\
\hline & Gestión para innovar $(\mathrm{G})$ & $\begin{array}{l}\text { Contabilización del número de tecnologías } \\
\text { instaladas en cada sistema }\end{array}$ \\
\hline \multirow[t]{4}{*}{ Ambiental } & $\begin{array}{l}\text { Contenido de carbono en } \\
\text { biomasa vegetal del estrato } \\
\text { superior }(\mathrm{C})\end{array}$ & $\begin{array}{l}\text { Metodología propuesta por Masuhara et al. (2015), } \\
\text { en donde se consideran ecuaciones alométricas para } \\
\text { cada especie arbórea y arbustiva }\end{array}$ \\
\hline & $\begin{array}{l}\text { Contenido de nitrógeno en } \\
\text { biomasa vegetal del estrato } \\
\text { inferior }(\mathrm{N})\end{array}$ & Se determinó por el método de Kjeldahl \\
\hline & $\begin{array}{l}\text { Biomasa de macrofauna } \\
\text { edáfica }(\mathrm{BM})\end{array}$ & $\begin{array}{l}\text { Se determinó en función del peso total de los } \\
\text { individuos por superficie }\left(\mathrm{m}^{2}\right) \text {, expresado en }\left(\mathrm{g} \mathrm{m}^{-2}\right)\end{array}$ \\
\hline & $\begin{array}{l}\text { Diversidad de macrofauna } \\
\text { edáfica (DM) }\end{array}$ & $\begin{array}{c}\text { Se determinó en función del número de especies de } \\
\text { organismos encontrados en cada uno de los } \\
\text { sistemas evaluados }\end{array}$ \\
\hline
\end{tabular}

Elaboración con información y toma de muestras del ciclo productivo 2018-2019.

Esta versión se distingue de su versión anterior (PROMETHEE I) en que genera un orden completo en la clasificación de las alternativas. El método contempla la estimación de tres componentes básicos que resultan de las comparaciones pareadas entre las distintas alternativas (Ishizaka y Nemery, 2013): grados de preferencia $\left(\mathrm{P}_{\mathrm{ij}}^{\mathrm{k}}\right)$, flujos unicriterio $\left(\frac{1}{\mathrm{n}-1} \sum_{\mathrm{j}=1}^{\mathrm{n}} \mathrm{P}_{1 \mathrm{n}}^{1}\right)$ y flujos globales. 
Los grados de preferencia $\left(\mathrm{P}_{\mathrm{ij}}^{\mathrm{k}}\right)$ expresan el grado en el cual la alternativa $a_{i}$ supera la alternativa $a_{j}$ en el criterio $k$. El método permite la posibilidad de enriquecer los grados de preferencia mediante el uso de funciones de preferencia. Brans and De Simet (2016) proponen seis funciones para expresar las preferencias. En este estudio se utiliza la función conocida como usual (Figura 1 y ecuaciones (1)), donde los valores de los umbrales de indiferencia y preferencia son igual a cero, lo que significa que ante la más mínima diferencia positiva e $f_{k}\left(a_{i}\right)-f_{k}\left(a_{j}\right) n$, el grado de preferencia es (fuerte) igual a 1. Si la diferencia resultara negativa o cero, el grado de preferencia es cero o indiferente. $P_{i j}^{k}=\left\{\begin{array}{ccc}0 & \text { si } & f_{k}\left(a_{i}\right)-f_{k}\left(a_{j}\right) \leq 0 \\ 1 & \text { si } & f_{k}\left(a_{i}\right)-f_{k}\left(a_{j}\right)>0\end{array}\right.$

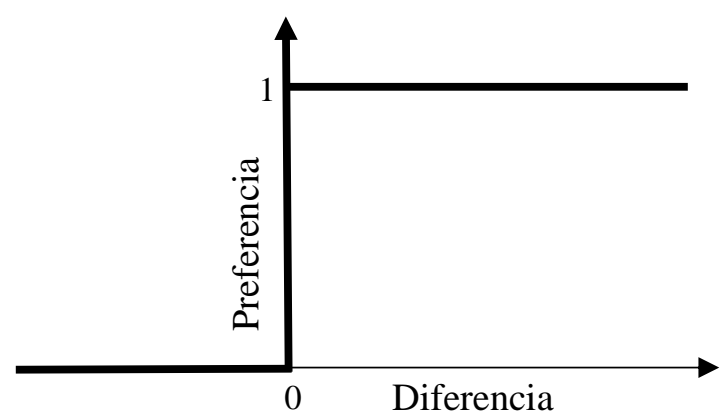

Figura 1. Función de preferencia usual (Brans and De Simet, 2016).

El flujo unicriterio es el promedio de los $\left(\mathrm{P}_{\mathrm{ij}}^{\mathrm{k}}\right)$ para cada alternativa en cada criterio. Se estiman en dos sentidos: el flujo unicriterio positivo $\left(\mathrm{FUP}=\frac{1}{\mathrm{n}-1} \sum_{\mathrm{j}=1}^{\mathrm{n}} \mathrm{P}_{1 \mathrm{n}}^{\mathrm{k}}\right)$ y el flujo unicriterio negativo $\left(\mathrm{FUN}=\frac{1}{\mathrm{n}-1} \sum_{\mathrm{i}=1}^{\mathrm{n}} \mathrm{P}_{\mathrm{n} 1}^{\mathrm{k}}\right)$. El FUP expresa cómo una alternativa es preferida sobre todas las demás en un criterio en particular. El FUN expresa cómo el resto de las alternativas son preferidas sobre una alternativa en particular. El flujo unicriterio neto (FUNeto) es la diferencia entre el FUP y el FUN. El flujo global toma en cuenta todos los criterios de manera simultánea, incluyendo sus pesos.

Se estiman el flujo global positivo $\left(\Phi^{+}\right)$, el flujo global negativo $\left(\Phi^{-}\right)$, y el flujo global neto $(\Phi)$. Este último determina la clasificación de las alternativas. Esto es, $\Phi^{+}=\mathrm{w}_{1}\left(\frac{1}{n-1} \sum_{\mathrm{j}=1}^{\mathrm{n}} \mathrm{P}_{\mathrm{ij}}^{1}\right)+\mathrm{w}_{2}\left(\frac{1}{\mathrm{n}-1} \sum_{\mathrm{j}=1}^{\mathrm{n}} \mathrm{P}_{\mathrm{ij}}^{2}\right)++\mathrm{w}_{\mathrm{n}}\left(\frac{1}{\mathrm{n}-1} \sum_{\mathrm{j}=1}^{\mathrm{n}} \mathrm{P}_{\mathrm{ij}}^{\mathrm{k}}\right) \Phi^{-}=\mathrm{w}_{1}\left(\frac{1}{\mathrm{n}-1} \sum_{\mathrm{i}=1}^{\mathrm{n}} \mathrm{P}_{\mathrm{ji}}^{1}\right)+$ $\mathrm{w}_{2}\left(\frac{1}{\mathrm{n}-1} \sum_{\mathrm{i}=1}^{\mathrm{n}} \mathrm{P}_{\mathrm{ji}}^{2}\right)++\mathrm{w}_{\mathrm{n}}\left(\frac{1}{\mathrm{n}-1} \sum_{\mathrm{i}=1}^{\mathrm{n}} \mathrm{P}_{\mathrm{ji}}^{\mathrm{k}}\right), \Phi=\Phi^{+}-\Phi^{-}$. Donde $\mathrm{w}_{\mathrm{i}}$ es el peso de importancia del criterio i.

La aplicación del método fue desarrollada utilizando el software Smart Picker Pro-versión 4.1.0. Finalmente, se analizó la estabilidad de los resultados obtenidos mediante el método PROMETHE, se realizaron distintos cambios en los valores de los criterios para los tres primeros sistemas de la clasificación resultante. 


\section{Resultados y discusión}

En los resultados del escenario financiero se observa claramente que el sistema con aguacate monocultivo (SAM) tiene la primera posición en la clasificación de los sistemas, con una RBC de 2.43, seguido por el sistema de aguacate con café (SAC) con una RBC de 2.36. Los sistemas restantes quedan a mayor distancia con RBC igual o menores a 1.3 (Cuadro 2). Esto da fundamento a la amenaza existente de que el monocultivo de aguacate, cuyo único criterio de decisión se basa en medidas monetarias, se establezca y sustituya a los otros sistemas que se caracterizan por incluir aspectos deseables y distintos a lo estrictamente financiero.

Estos aspectos deseables, como la diversificación de cultivos y especies, el manejo de tecnologías amigables con el ambiente, la aplicación de vermicomposta, y consideraciones sociales son características de los sistemas agroforestales con café, clasificados según Escamilla (1994) como policultivos tradicionales.

En el análisis del escenario multicriterio, la determinación de los distintos desempeños de los sistemas en los diferentes criterios incluidos configura una matriz de decisión con las características clásicas de los problemas multicriterio, esto es, ninguno de los sistemas presenta los mejores desempeños en todos los criterios (Cuadro 2). Del mismo modo, tampoco alguno de ellos presenta los peores valores en todos los criterios. Esto presenta parte de la complejidad en el proceso de priorización de este tipo de alternativas, además de evidenciar que los valores de los desempeños de los criterios permitirán identificar las ventajas y desventajas de cada sistema evaluado (Bengtsson y Steen, 2004).

Cuadro 2. Matriz de desempeños de los sistemas en cada criterio.

\begin{tabular}{ccccccccc}
\hline Sistema & RBC & NI & E & G & C & N & BM & DM \\
\hline SCA & 0.96 & 18750 & 6.98 & 1 & 41.84 & 1.61 & 149.67 & 8 \\
SCR & 1.3 & 45660 & 75.33 & 2 & 40.79 & 1.17 & 124 & 5 \\
SCL & 1.06 & 31740 & 114.72 & 1 & 40.04 & 1.01 & 118.11 & 7 \\
SAC & 2.36 & 41310 & 70.99 & 2 & 38.94 & 0.05 & 1.67 & 2 \\
SAM & 2.43 & 27600 & 38.43 & 2 & 26.25 & 0.03 & 10 & 1 \\
\hline
\end{tabular}

Elaboración con información y toma de muestras del ciclo productivo 2018-2019.

Para la estimación de la clasificación del segundo escenario caracterizado por la inclusión adicional de criterios sociales y ambientales, mediante la aplicación del PROMETHEE, se calcularon los grados de preferencia a partir de los cuales se computaron los flujos netos unicriterio (Cuadro 3).

Cuadro 3. Flujos unicriterio netos.

\begin{tabular}{ccccccccc}
\hline Sistemas & RBC & NI & E & G & C & N & BM & DM \\
\hline SCA & -1 & 1 & -1 & -0.75 & 1 & 1 & 1 & 1 \\
SCR & 0 & -1 & 0.5 & 0.5 & 0.5 & 0.5 & 0.5 & 0 \\
SCL & -0.5 & 0 & 1 & -0.75 & 0 & 0 & 0 & 0.5 \\
SAC & 0.5 & -0.5 & 0 & 0.5 & -0.5 & -0.5 & -0.5 & -0.5 \\
SAM & 1 & 0.5 & -0.5 & 0.5 & -1 & -1 & -1 & -1 \\
\hline
\end{tabular}


De la información presente en el Cuadro 3 se destaca que solo los sistemas aguacate monocultivo (SAM) y aguacate café (SAC) presentan valores de flujo unicriterio neto positivos en la dimensión financiera (RBC), adicionalmente, ambos presentan valores de flujo unicriterio neto negativos en todos los criterios ambientales, evidenciando con ello que el manejo agronómico intensivo de los sistemas afecta negativamente el mantenimiento de los recursos en los criterios ambientales.

Por su parte, el resto de los sistemas, SCA, SCR y SCL presentan valores positivos unicriterio en los criterios relacionados a macrofauna del suelo, esto se puede atribuir al reciclaje de residuos orgánicos que se genera en ambos sistemas producto de la alta densidad de siembra y las podas severas, que generan residuos orgánicos y condiciones edafoclimáticas que contribuyen a la actividad de la macrofauna del suelo (Paolini, 2018). Además, reportaron flujo positivo en los contenidos de $\mathrm{C}$ y $\mathrm{N}$, por tanto, se confirma que los sistemas diversificados contribuyen a los almacenes de carbono y disponibilidad de nitrógeno en el suelo (Gallardo, 2017).

Dado que los flujos unicriterio neto son una medida de la preferencia que se tiene por uno de los sistemas en particular, en el criterio en cuestión, sobre el resto de los sistemas, esta información nos adelanta las fortalezas y debilidades de cada sistema en cada criterio, lo que posteriormente habrá de definir la prioridad, en función de los pesos de importancia asignados a cada uno de los criterios. Posteriormente se estimaron los flujos globales netos, mismos que resultan de la suma ponderada de dichos flujos unicriterio para cada sistema y a su vez, estos definen la clasificación de los sistemas (Cuadro 4).

La aplicación del método PROMETHEE generó una clasificación distinta del escenario financiero. Sobresale el ascenso a la primera posición del sistema de café renovado con variedades tolerantes a roya (SCR). El sistema de aguacate monocultivo (SAM) pasó, de la primera, a una segunda posición. Los sistemas, aguacate con café (SAC) y el sistema café con podas de limpieza severa (SCL), perdieron una posición para ocupar la tercera y quinta posición, respectivamente, en la clasificación. El sistema de café con abandono de cultivo por 12 años (SCA) avanzó un lugar para quedar en la cuarta posición (Cuadro 4).

Cuadro 4. Clasificación de los escenarios financiero y multicriterio.

\begin{tabular}{cccccc}
\hline Sistemas & RBC & $\begin{array}{c}\text { Clasificación } \\
\text { RBC }\end{array}$ & $\begin{array}{c}\text { Flujo global } \\
\text { neto }\end{array}$ & $\begin{array}{c}\text { Clasificación } \\
\text { PROMETHEE }\end{array}$ & Variación en la posición \\
\hline SAM & 2.43 & 1 & 0.05534 & 2 & -1 \\
SAC & 2.36 & 2 & -0.0001 & 3 & -1 \\
SCR & 1.3 & 3 & 0.12505 & 1 & +2 \\
SCL & 1.06 & 4 & -0.09717 & 5 & -1 \\
SCA & 0.96 & 5 & -0.08311 & 4 & +1 \\
\hline
\end{tabular}

En la Figura 2 se presenta gráficamente la clasificación de los sistemas correspondientes al escenario multicriterio. El orden de mayor a menor prioridad va de izquierda a derecha, y las aportaciones de los criterios se muestran en rectángulos de distintos colores que cubren una escala entre -1 y +1 . De acuerdo con los resultados, el mejor evaluado es el sistema con café tolerante a roya (SCR). El valor de su flujo global neto (0.125, en Cuadro 4), resulta de la fortaleza en los criterios empleo $(E)$ y gestión para innovar $(G)$ cuyos valores de desempeño (75.33 jornales y 2 
tecnologías, respectivamente, Cuadro 2), permitieron que en las comparaciones pareadas con el resto de los sistemas obtuviera flujos unicriterio netos positivos ( 0.5 en ambos, Cuadro 3), mismos que al multiplicarse por los respectivos pesos (0.111 en ambos) generaron aportes positivos en la sumatoria que del flujo global neto (0.056 en ambos, Figura 2).

Una condición similar se presenta en los desempeños de los criterios C, N y BM, solo que en este caso los tres pertenecen a la dimensión ambiental y el peso de importancia asignado a cada uno es de 0.0834 , de modo que el aporte positivo de cada criterio es un poco menor (0.042, Figura 2). El caso del criterio RBC tiene un aporte cero al Flujo global neto de SCR. Esto se explica porque su desempeño en este criterio (1.3, en Cuadro 2) corresponde al punto medio entre los cinco sistemas.

Esto es, al compararse en forma pareada con los otros sistemas, resulta que domina a dos de ellos y otros dos le dominan. Esto genera que el flujo neto unicriterio del sistema, en el criterio RBC, sea cero (Cuadro 3). El criterio necesidad de inversión (NI) presenta un aporte negativo al flujo global neto del sistema. Esto obedece a que SCR tiene la necesidad de inversión mayor que el resto de los sistemas ( $\$ 45660.00$, Cuadro 2) y como es un criterio a minimizar, entonces el resto de los sistemas tienen valores preferibles que dan como resultado un flujo neto unicriterio de -1 (Cuadro 3), mismo que al multiplicarse por su peso (0.111), genera un aporte negativo de -0.111 (Figura 2).

Un análisis similar se puede hacer en las causales del ordenamiento en el resto de los sistemas aquí considerados. En el sistema que ocupa la segunda posición, aguacate monocultivo (SAM), el criterio de mayor aporte es la RBC (0.333), lo cual resulta porque SAM es superior al resto de los sistemas en este criterio. Sin embargo, hay cinco criterios cuyos aportes le son negativos. Por tanto, estos criterios deben considerarse para promover la diversificación productiva de los cafetales, puesto que la capacidad de inversión del pequeño productor influirá en la programación y decisión de implementar las alternativas de manejo agronómico (Martinelli et al., 2019), que repercutirá en el éxito de la producción e ingreso del sistema como alternativa al monocultivo de aguacate.

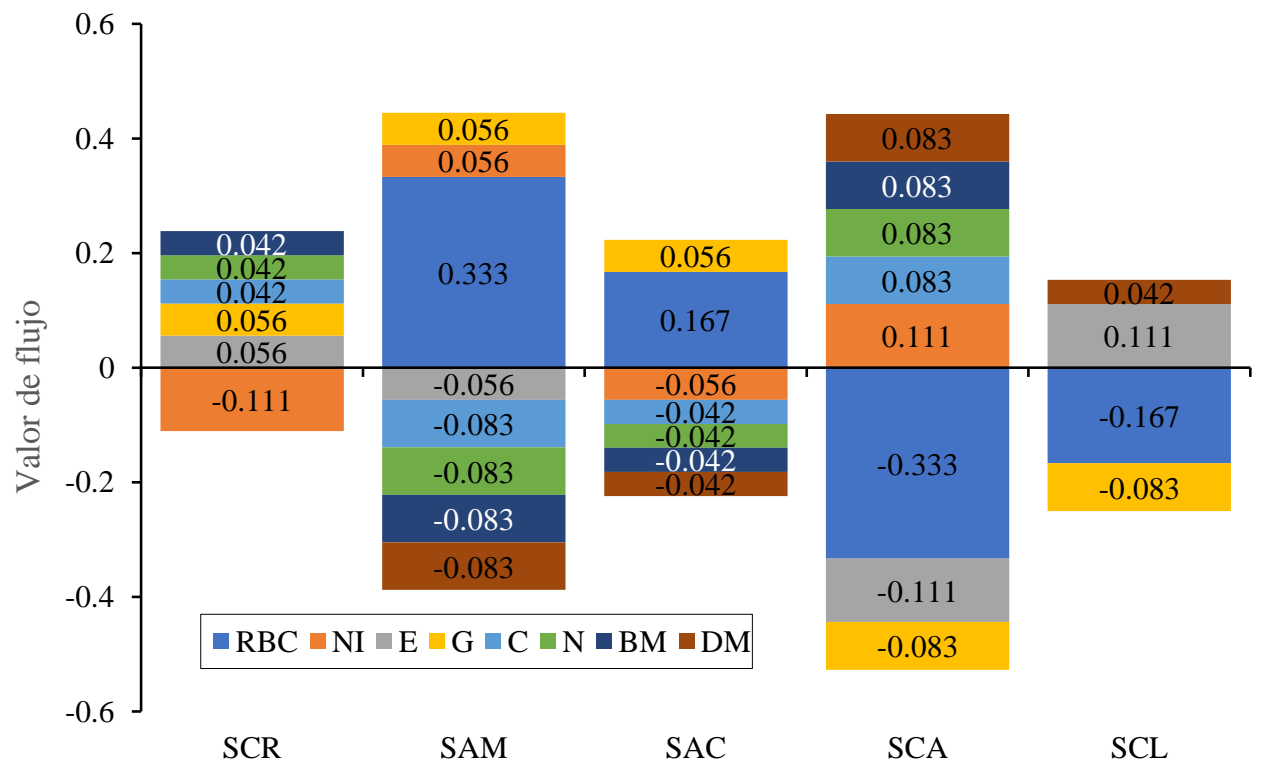

Figura 2. Clasificación de los sistemas incluyendo aportes de los criterios. 
Finalmente, y con la idea de analizar la estabilidad de estos resultados, se hicieron modificaciones en los valores de los desempeños de los sistemas posicionados en los tres primeros lugares de la clasificación: sistema de café tolerante a roya (SCR), sistema de aguacate monocultivo (SAM) y sistema de aguacate y café (SAC). El análisis consistió en estimar el valor crítico de cada criterio en los tres sistemas, entendido éste como el mínimo cambio en el criterio, manteniendo los demás valores constantes, que causa una modificación en la posición del sistema en la clasificación (Triantaphyllou, 2000), resultados que confirman que el criterio RBC en el SAM se debe a los ingresos económicos que genera por el cultivo de aguacate (Franco et al., 2018). Para el SCR, las variaciones en los valores de los criterios se hicieron a la baja con el fin de identificar el punto en que este sistema perdía su primera posición, para los otros dos sistemas (SAM y SAC), las variaciones fueron al alza, para observar el punto en que ascendían su posición, excepto en NI cuya variación fue a la baja por ser un criterio para minimizar.

Los resultados obtenidos indican que este ordenamiento generado por el método PROMETHEE es bastante estable puesto que en la mayor parte de los criterios se requieren variaciones demasiado grandes para provocar un cambio en el mismo (Cuadro 5). Aunque en el caso de $\mathrm{C}$ en SCR y en $\mathrm{SAC}$, los porcentajes podrían parecer menores, en realidad no pues se debe tomar en cuenta que un incremento de 1\% en contenido de carbón en el sistema puede tomar varios años (Gallardo, 2017).

Cuadro 5. Sensibilidad de la clasificación resultante del método PROMETHE.

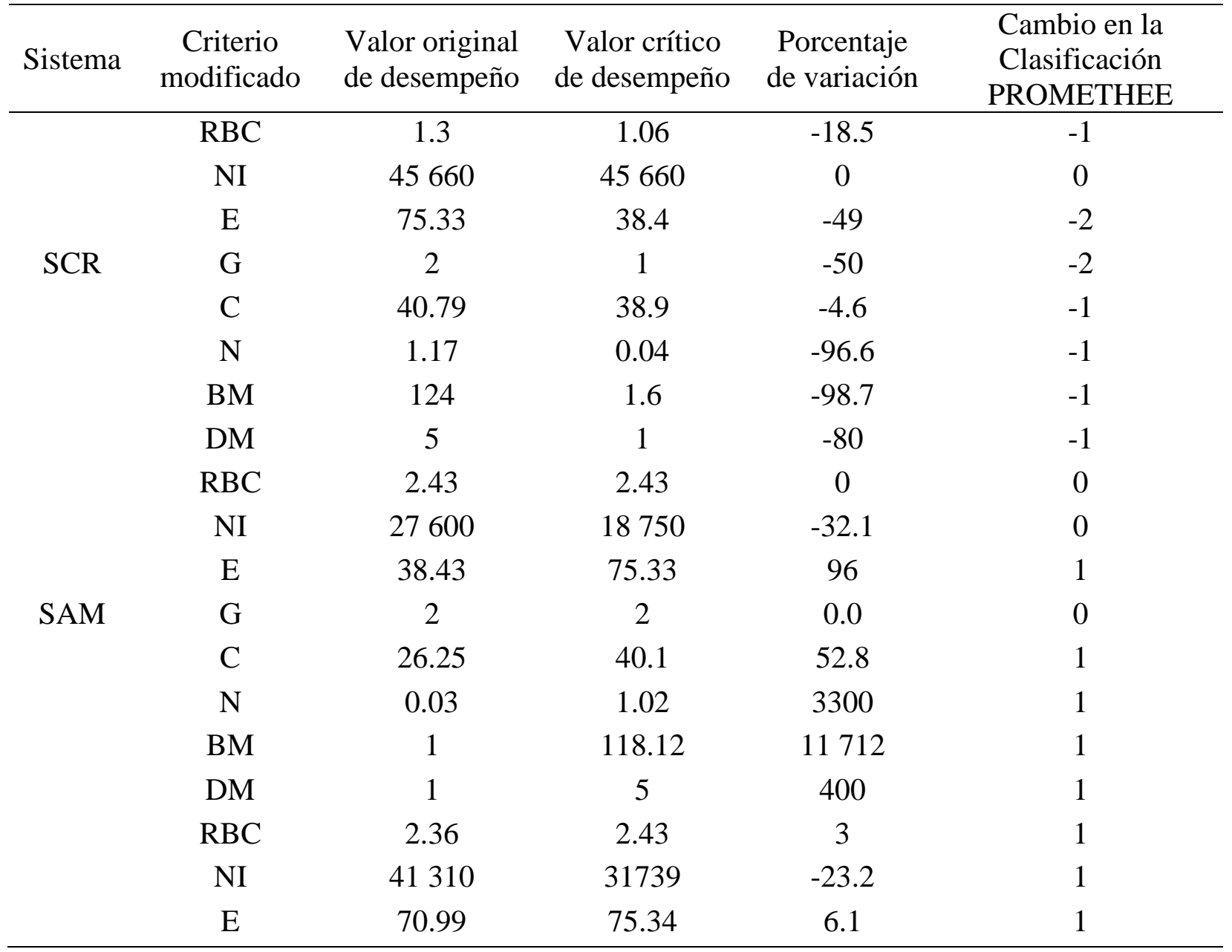




\begin{tabular}{cccccc}
\hline Sistema & $\begin{array}{c}\text { Criterio } \\
\text { modificado }\end{array}$ & $\begin{array}{c}\text { Valor original } \\
\text { de desempeño }\end{array}$ & $\begin{array}{c}\text { Valor crítico } \\
\text { de desempeño }\end{array}$ & $\begin{array}{c}\text { Porcentaje } \\
\text { de variación }\end{array}$ & $\begin{array}{c}\text { Cambio en la } \\
\text { Clasificación } \\
\text { PROMETHEE }\end{array}$ \\
\hline SAC & G & 2 & 2 & 0 & 0 \\
& $\mathrm{C}$ & 38.94 & 40.79 & 4.8 & 1 \\
& $\mathrm{~N}$ & 0.05 & 1.17 & 2240 & 1 \\
& $\mathrm{BM}$ & 1.67 & 124 & 7325.1 & 1 \\
& $\mathrm{DM}$ & 2 & 7 & 250 & 1 \\
\hline
\end{tabular}

En el caso de los porcentajes de variación cero (NI en SCR, RBC en SAM y G en SAC), no se realizaron modificaciones porque no causarían el efecto buscado. NI, como criterio para minimizar, en SCR ya es el peor valor entre los sistemas, de modo que ningún incremento en éste modificaría su primera posición. Los casos de RBC y G son similares, pero en sentido contrario, ambos son a maximizar y tienen el valor máximo entre los sistemas, de modo que no hay incrementos que mejores su posición de sus sistemas en la clasificación. Estos resultados muestran deficiencia en la RBC para el sistema SCR, por tanto, es necesario la diversificación productiva con otro cultivo para contribuir a la priorización de sistemas agroforestales productivos y con mejores ingresos económicos (Jezeer et al., 2018).

\section{Conclusiones}

Los análisis realizados dan fundamento a la amenaza presente de desplazamiento de sistemas tradicionales de producción asociados al café por sistemas de aguacate monocultivo. Por una parte, el análisis tradicional basado solo en medidas monetarias, análisis financiero, demuestra que la rentabilidad financiera obtenida en el SAM es muy superior a la obtenida en los otros sistemas. Asimismo, en el análisis multicriterio, aun cuando se consideran variables de interés social y ambiental, el SAM mantiene una segunda posición en el orden de prioridad.

En estos resultados, además de las valoraciones no monetarias, influyen de manera importante los pesos de importancia asignados a los distintos criterios considerados, de este modo, ante un escenario en el que los tomadores de decisiones de políticas públicas expresaran un mayor interés por las variables sociales y ambientales, se podrían asignar pesos mayores a éstas y así consolidar los sistemas que asocian café y aguacate en mejores posiciones de la clasificación.

Aun así, se mantiene el reto de diseñar y poner en práctica políticas públicas que incentiven tales sistemas que evidentemente son más sostenibles en términos sociales y ambientales.

\section{Literatura citada}

Aguirre, J. F.; Cadena, J. y Ramírez, B. 2016. Diversificación de cultivos en fincas cafetaleras como estrategia de desarrollo. Caso de Amatlán. Acta Universitaria. 26(1):30-38. Doi:10.15174/ au.2016.833.

Ayala, D.; Monterroso, A. I.; Baca, J.; Escamilla, E.; Sánchez, R.; Pérez, N.; Rajagopal, I.; Alegre, J. C. y Valdés, E. 2020. Identificación de necesidades de investigación sobre la dinámica de carbono y nitrógeno en sistemas agroforestales de café en México. Trop. Subtrop. Agroecosystems. 23(2020):99. 
Bengtsson, M. and Steen, B. 2004. Weighting in LCA-approaches and applications. Environmental Progress. 19(4):101-109. https://doi.org/10.1002/ep.670190208.

Benoit, V. and Rousseaux, P. 2003. Aid for aggregating the impacts in Life Cycle assessment. Inter. J. Life Cycle Assessment. 8(2):74-89. https://doi.org/10.1007/ BF0297843.

Berbel, J.; Bournaris, T.; Manos, B.; Matsatsinis, N. and Viaggi, D. 2018. Multicriteria analysis in agriculture: current trends and recent applications. In: Berbel, J.; Bournaris, T.; Manos, B.; Matsatsinis, N. and Viaggi, D. (Ed.). Frist edition. Springer. Switzerland. 328 p.

Bournaris, T. and Manos, B. 2012. European union agricultural policy scenarios' impacts on social sustainability of agricultural holdings. Inter. J. Sustainable Development \& World Ecology. 19(5):426-432. https://doi.org/10.1080/13504509.2012.670670.

Bournaris, T.; Moulogianni, C. and Manos, B. 2014. A multicriteria model for the assessment of rural development plans in Greece. Land Use Policy. 38(3):1-8. https://doi.org/10.1016/j.landusepol.2013.10.008.

Bournaris, T.; Papathanasiou, J.; Moulogianni, C. and Manos, B. 2009. A fuzzy multicriteria mathematical programming model for planning agricultural regions. New Medit. 8(4):22-27.

Brans, J. P. and De Smet, Y. 2016. Promethee methods. In: multiple criteria decision analysis: state of the art surveys. Springer (Ed.). Firth edition. New York, USA. 195 p. Doi: 10.1007/9781-4939-3094-4_6.

Caprara, C. and Martelli, R. 2016. Multi-criteria analysis of suitability for energy crops under structural and environmental constraints: a case study in a northeastern Italian region. Transactions of the ASABE. 59(4):815-828. Doi: 10.13031/trans.59.11229.

Castellini, C.; Boggia, A.; Cortina, C.; Dal Bosco, A.; Paolotti, L.; Novelli, E. and Mugnai, C. 2012. A multicriteria approach for measuring the sustainability of different poultry production systems. J. Cleaner Production. 37(4):192-201. https://doi.org/10.1016/j.jclepro.2012.07.006.

De Luca, A. I.; Iofrida, N.; Leskinen, P.; Stillitano, T.; Falcone, G.; Strano, A. and Gulisano, G. 2017. Life cycle tools combined with multi-criteria and participatory methods for agricultural sustainability: Insights from a systematic and critical review. Science of the Total Environment. 595(1):352-370. https://doi.org/10.1016/j.scitotenv.2017.03.284.

De Luca, A. I.; Iofrida, N.; Strano, A.; Falcone, G. and Gulisano, G. 2015. Social life cycle assessment and participatory approaches: a methodological proposal applied to citrus farming in Southern Italy. Integrated Environmental Assessment and Management. 11(3):383-396. https://doi.org/10.1002/ieam.1611

Dinh, L. T. T.; Guo, Y. and Mannan, M. S. 2009. Sustainability evaluation of biodiesel production using multicriteria decision-making. Environmental Progress Sustainable Energy. 28(1):38-46. https://doi.org/10.1002/ep.10335.

Dussán, L. C.; Duque, O. H.; González, L. J. 2006. Caracterización tecnológica de caficultores de economía campesina, de los principales municipios cafeteros de Colombia. Cenicafé. 57(3):167-186.

Escalante, M. y Somarriba, E. 2001. Diseño y manejo de los cafetales del Occidente de El Salvador. CATIE, Turrialba, Costa Rica. Agroforestería en las Américas. 8(30):12-16. http://hdl.handle.net/11554/6061.

Escamilla, E.; Licona, A. L.; Díaz, S.; Sontoyo, H. V.; Sosa, R. y Rodríguez, L. 1994. Los sistemas de producción de café en el centro de Veracruz, México. Un análisis tecnológico. Revista de Historia. UNA 30(2):41-67. 
Falcone, G.; De Luca, A.; Stillitano, T.; Strano, A.; Romeo, G. and Gulisano, G. 2016. Assessment of environmental and economic impacts of vine-growing combining life cycle assessment, life cycle costing and multicriterial analysis. Sustainability. 8(8):793-827. https://doi.org/10.3390/ su8080793

Finkbeiner, M.; Schau, E. M.; Lehmann, A. and Traverso, M. 2010. Towards life cycle sustainability assessment. Sustainability. 10(2):3309-3322. https://doi.org/10.3390/ su2103309.

FIRA. 2016. Fideicomisos Instituidos en Relación con la Agricultura. Panorama agroalimentario. Dirección de investigación y evaluación económica y sectorial. Café 2016. Documento técnico de Fideicomisos Instituidos en Relación con la Agricultura. México. 37 p.

Franco, M.; Leos, J.; Salas, J.; Acosta, M. y García, A. 2018. Análisis de costos y competitividad e la producción de aguacate en Michoacán, México. Rev. Mex. Cienc. Agríc. 9(2):391-403. http://dx.doi.org/10.29312/remexca.v9i2.1080.

Gallardo, J. F. 2017. La materia orgánica del suelo, residuos orgánicos, humus, compostaje y captura de carbono. Gallardo, J. F. (Ed.). Universidad Autónoma Chapingo (UACH). Chapingo, Estado de México. 424 p.

Granados, R.; Medina, Ma de la Paz y Peña, V. 2014. Variación y cambio climático en la vertiente del Golfo de México: impactos en la cafeticultura. Rev. Mex. Cienc. Agríc. 5(3):473-485.

Hermann, B. G.; Kroeze, C. and Jawjit, W. 2007. Assessing environmental performance by combining life cycle assessment, multi-criteria analysis and environmental performance indicators. J. Cleaner Production. 15(18):1787-1796. https://doi.org/10. 1016/j.jclepro.2006.04.004.

Ishizaka, A. and Nemery, P. 2013. Multi-criteria decision analysis: methods and software. Wiley, J. \& Sons (Ed). Frist edition. Chichester, West Sussex, PO 19 8SQ, United Kingdom. 296 p. doi:10.1002/9781118644898.

Jezeer, R. E; Santos, M. J.; Bota, G. A.; Junginger, M. and Verweij, P. A. 2018. Effects of shade and input management on economic performance of small-scale Peruvian coffee systems. Agricultural Systems. 162(4):179-190 pp. Doi: 10.1016/j.agsy.2018.01.014.

Karklina, K.; Slisane, D.; Romagnoli, F. and Blumberga, D. 2015. Social life cycle assessment of biomethane production and distribution in Latvia. Environment. Technology. Resources. 2(2):128-132. Proceedings of the $10^{\text {th }}$ International Scientific and Practical Conference, Rezekne, Latvia. https://doi.org/10.17770/etr2015vol2.628.

Kralisch, D.; Staffel, C.; Ott, D.; Bensaid, S.; Saracco, G.; Bellantoni, P. and Loeb, P. 2013. Process design accompanying life cycle management and risk analysis as a decision support tool for sustainable biodiesel production. Green Chemistry. 15(2):463-477. https://doi.org/10.1039/C2GC36410G.

Kylili, A.; Christoforou, E.; Fokaides, P. A. and Polycarpou, P. 2016. Multicriteria analysis for the selection of the most appropriate energy crops: the case of cyprus. Inter. J. Sustainable Energy. 35(1):47-58. https://doi.org/10.1080/14786451.2014.898640.

Leiva, S. T.; Román, A. y Rubio, K. B. 2017. Caracterización socioeconómica de la producción cafetalera en la provincia de Rodríguez de Mendoza (Amazonas, Perú). Agroproducción Sustentable. 1(3):59-67. Doi: 10.25127/aps.20173.375.

Lipuscek, I.; Bohanec, M.; Oblak, L. and Zadnik, S. L. 2010. A multi-criteria decision-making model for classifying wood products with respect to their impact on environment. Inter. J. Life Cycle Assessment. 15(4):359-367. https://doi.org/10.1007/s11367-010-0157-6. 
Maia, A. A. C.; Saraiva, A. B.; Clímaco, J. C. N.; Infante, C. E. and Valle, R. 2017. Life cycle assessment and multi-criteria decision analysis: Selection of a strategy for domestic food waste management in Rio de Janeiro. J. Cleaner Production. 143(1):744-756. https://doi.org/10.1016/j.jclepro.2016.12.049.

Manos, B.; Bournaris, T.; Chatzinikolaou, P.; Berbel, J. and Nikolov, D. 2013. Effects of CAP policy on farm household behaviour and social sustainability. Land Use Policy. 31:166-181. https://doi.org/10.1016/j.landusepol.2011.12.012.

Manos, B.; Bournaris, T.; Kamruzzaman, M.; Begum, M.; Anjuman, A. and Papathanasiou, J. 2006. Regional impact of irrigation water pricing in Greece under alternative scenarios of European policy: A multicriteria analysis. Regional Studies. 40(9):1055-1068. https://doi.org/10.1080/00343400600928335.

Manos, B.; Papathanasiou, J.; Bournaris, T. and Voudouris, K. 2010. A multicriteria model for planning agricultural regions within a context of groundwater rational management. J. Environmental Management. 91(7):1593-1600. https://doi.org/10.1016/j.jenvman. 2010.03.002.

Martinelli, G. do C.; Schlindwein, M. M.; Padovan, M. P. and Gimenes, R. M. T. 2019. Decreasing uncertainties and reversing paradigms on the economic performance of agroforestry systems in Brazil. Land Use Polic. 80(1):274-286.

Moffett, A. and Sarkar, S. 2006. Incorporating multiple criteria into the design of conservation area networks: a mini review with recommendations. Diversity and Distributions. Diversity Distrib. 12(2):125-137. https://doi.org/10.1111/j.1366-9516.2005.00202.x.

Myllyviita, T.; Holma, A.; Antikainen, R.; Lähtinen, K. and Leskinen, P. 2012. Assessing environmental impacts of biomass production chains-application of life cycle assessment (LCA) and multi-criteria decision analysis (MCDA). J. Cleaner Production. 29(10):238-245. ISSN: 0959-6526. https://doi.org/10.1016/j.jclepro.2012.01.019.

Myllyviita, T.; Leskinen, P. and Seppälä, J. 2014. Impact of normalization, elicitation technique and background information on panel weighting results in life cycle assessment. Inter. J. Life Cycle Assessment. 19(2):377-386. https://doi.org/10.1007/s11367-013-0645-6.

Paolini, J. E. 2018. Actividad microbiológica y biomasa microbiana en suelos cafetaleros de los Andes venezolanos. Terra Latinoam. 36(1):13-22. Doi: https://doi.org/10.28940/ terra.v36i1.257.

Pérez, V. y Garza, G. 2013. Evaluación de la sustentabilidad del sistema de manejo de café orgánico en la unión de ejidos Majomut, región de los Altos de Chiapas. In: Masera, O. y López, S. (Ed). Sustentabilidad y sistemas campesinos: cinco experiencias de evaluación en el México rural. Mundi-Prensa México. 45-81.

Pertuz, V. P. y Pérez, A. B. 2017. Caracterización socioeconómica de los productores de cafés especiales de Pueblo Bello (Cesar, Colombia). Punto de Vista. 8(11):117-140.

Popescu, G. and Bara, S. 2015. Regional development disparities in Romanian agriculture and rural development: a multi-criteria approach. In: Popescu, G. and Jean-Vasile, A. (Ed.). Agricultural management strategies in a changing economy. Hershey, P. A. IGI Global. 29 p. https://doi.org/10.4018/978-1-4666-7521-6.ch001.

Riesgo, L. and Gómez, J. A. 2006. Multi-criteria policy scenario analysis for public regulation of irrigated agriculture. Agricultural Systems. 91(1-2):1-28. https://doi.org/10.1016/j.agsy. 2006.01.005.

Sánchez, S.; Mendoza, M. A. y García, R. V. 2017. Diversificación de la sombra tradicional de cafetales en Veracruz mediante especies maderables. Rev. Mex. Cienc. Forest. 8(40):7-17. https://doi.org/10.29298/rmcf.v8i40.32. 
Sánchez-Hernández, S.; Escamilla-Prado, E.; Mendoza-Briseño, M. A. y Nazario-Lezama, N. 2018. Calidad del café (Coffea arabica L.) en dos sistemas agroforestales en el centro de Veracruz, México. AgroProductividad. 11(4):80-86.

Triantaphyllou, E. 2000. A sensitivity analysis approach for MCDM Methods. In: multi-criteria decision-making methods: a comparative study. Applied optimization. Springer (Ed). Firth edition. Boston, MA. Vol. 44. 290 p. Doi: 10.1007/978-1-4757-3157-6.

Tziolas, E.; Manos, B. and Bournaris, T. 2017. Planning of agro-energy districts for optimum farm income and biomass energy from crops residues. Operational Research. 17(2):535-546. https://doi.org/10.1007/s12351-016-0236-y.

Wu, Y.; Yan, Y.; Wang, S.; Liu, F.; Xu, C. and Zhang, T. 2019. Study on location decision framework of agroforestry biomass cogeneration project: a case of China. Biomass and Bioenergy. 127(8):1-12. https://doi.org/10.1016/j.biombioe.2019.105289. 